\title{
Terahertz Time-domain Spectroscopy as a Novel Tool for Crystallographic Analysis in Cellulose: the Potentiality of Being a New Standard for Evaluating Crystallinity
}

Han Wang ( $\square$ wang.han@f.mbox.nagoya-u.ac.jp)

Nagoya University: Nagoya Daigaku https://orcid.org/0000-0001-5071-2020

Satoru Tsuchikawa

Nagoya University: Nagoya Daigaku

Tetsuya Inagaki

Nagoya University: Nagoya Daigaku

\section{Research Article}

Keywords: THz-TDS, XRD, Cellulose, Crystallinity, MCC, Wood

Posted Date: March 17th, 2021

DOl: https://doi.org/10.21203/rs.3.rs-291523/v1

License: (c) (i) This work is licensed under a Creative Commons Attribution 4.0 International License.

Read Full License 


\title{
Terahertz time-domain spectroscopy as a novel tool for crystallographic analysis in cellulose: the potentiality of being a new standard for evaluating crystallinity
}

\author{
Han Wang†, Satoru Tsuchikawa†, Tetsuya Inagaki†* \\ †raduate School of Bioagricultural Sciences, Nagoya University, Nagoya, Aichi, \\ 464-8601, Japan. \\ *E-mail: inatetsu@agr.nagoya-u.ac.jp \\ ABSTRACT
}

Given that terahertz $(\mathrm{THz})$ radiation responds to intermolecular forces such as hydrogen bonds, $\mathrm{THz}$ time-domain spectroscopy (THz-TDS) has expanded possibilities in cellulose research. In this study, THz-TDS was used to investigate the crystallinity of three types of cellulose-based materials.

Microcrystalline cellulose (MCC) and wood were ball milled at different times, and pseudo-wood was a mixture of MCC and lignin of different mass fractions. All the samples showed peaks at $3.04 \mathrm{THz}$ in the THz mass absorption coefficient spectra. Further, the spectra from $2.79 \mathrm{THz}$ to $3.32 \mathrm{THz}$ were cut out and detrended by subtraction from a baseline. The integrated intensity of the detrended spectra showed a correlation with the mass fraction of lignin of the pseudo-wood samples, and ball milling time of the MCC and wood samples. The correlation was similar with the crystallinity index calculated from X-ray powder diffraction. Moreover, the original wood sample without ball milling had an integrated intensity that was about $30 \%$ that of the original MCC sample, matching with the cellulose concentration of the wood (about $30 \%$ to $40 \%$ ). We normalized the integrated intensity of $2.79 \mathrm{THz}$ to $3.32 \mathrm{THz}$ into 1 to 0 by a min-max algorithm and proposed a new "index" for evaluating crystallinity.

Keywords: THz-TDS, XRD, Cellulose, Crystallinity, MCC, Wood Declarations.

Funding: JSPS KAKENHI Grant Number 16H02559

Conflicts of interest/Competing interests: None

Availability of data and material: None 
Code availability: Matlab $2018 \mathrm{~b}$

Authors' contributions: Han Wang and Tetsuya Inagaki conceived and designed the experiments. Han Wang performed the experiments, analyzed the data and wrote the paper, Tetsuya Inagaki and Satoru Tsuchikawa gave final approval of the manuscript. 


\section{Introduction}

2 Cellulose is a macromolecular polysaccharide composed of glucose units, where the $\beta(1 \rightarrow 4)$ -

3 D-glucose units are linked to a linear chain by hydrogen bonds (Updegraff 1969) and the cellulose

4 chains further are linked to a sheet structure. The abundant intra- and inter-chain hydrogen bonds in

5 cellulose make its structure relatively stable (Nishiyama et al. 2002; Langan et al. 2005). The sheets

6 of cellulose are spontaneously and orderly packed by hydrophobic forces such as van der Waals

7 forces, and further aggregated into larger microfibrils (Nishiyama 2009). The degree of order of

8 cellulose crystals can be evaluated in general by using the crystallinity index (CrI), which is an

9 important property of cellulose, and measured by X-ray powder diffraction (XRD). Cellulose is

10 abundantly contained in the cell walls of plants and algae and plays a role in supporting cell

11 structure. Wood is a typical natural cellulose-based material with a wide application and long

12 history. The CrI of cellulose reflects the physical and chemical properties of wood to a certain extent.

13 The tensile strength, Young's modulus, density, thermal stability, and dimensional stability of wood

14 all increase as the cellulose CrI increases (Density et al. 1975; Thygesen et al. 2005; Poletto et al.

15 2012), whereas the swelling and reaction speed of enzymatic conversion processes decrease

16 (Jörgensen et al. 1950; Hall et al. 2010). This condition means that crystalline cellulose is one of the

17 main rate-limiting barriers for the efficient transformation and utilization of plant biomass (Himmel

18 et al. 2007). Therefore, the crystallinity of cellulose must be investigated.

19 In addition to XRD, the methods for investigating the crystallinity of cellulose include solid-state carbon 13 nuclear magnetic resonance (13 $\mathrm{C}$ NMR), cross polarization/magic angle spinning

21 (CP/MAS), infrared spectroscopy (IR), near-infrared spectroscopy (NIR), and Raman spectroscopy

22 (Teeäär et al. 1987; Kataoka and Kondo 1998; Inagaki et al. 2010; Kim et al. 2013). Among these methods, XRD is the most widely used and considered as a standard method for evaluating crystallinity in most cases given that its pattern reflects the arrangement of atoms, and it has a rigorous theoretical calculation formula. However, the CrI obtained by XRD will be significantly different depending on the selected calculation method. The most used methods for determining the 
minimum diffraction intensity between the (200) and (110) crystalline diffraction peaks usually

appears at about $18^{\circ}$, where was attributed to amorphous by Segal et al., however, the diffraction

intensity at this position overlaps the crystalline intensity peaks; thus, the reliability of the first two methods is questionable (French and Santiago Cintrón 2013; French 2014; Ling et al. 2019).

Therefore, the peak deconvolution method was most discussed in recent studies. For the deconvolution process, the most used peak profiles are Gaussian, Lorentzian, Voigt, and pseudoVoigt (Hindeleh and Johnson 1972; de Keijser et al. 1983; Wada et al. 1997). However, the accurate expression of amorphous curves using only such functions is difficult (del Cerro et al. 2020). Yao et al. reported an amorphous curve fitted using Fourier series modeling, which gave a result that was consistent with the measured amorphous cellulose pattern (Yao et al. 2020).

The terahertz (THz) band has received considerable attention; this band is located in the farinfrared region where the boundary area is between light and radio waves, with a frequency range of $0.1 \mathrm{THz}$ to $10 \mathrm{THz}$, corresponding to wavelengths of $3 \mathrm{~mm}$ to $0.03 \mathrm{~mm}$. The earliest commercial employed application of this band was THz time-domain spectroscopy (THz-TDS). The THz band responds to the intermolecular vibration and rotation of numerous biological macromolecules; thus, the THz band has been used in biology (Plusquellic et al. 2007; Markelz 2008), biomaterials (Inagaki et al. 2014a, b; Wang et al. 2019), hydrodynamics (Braly et al. 2000), and medicine studies (Xie et al. 2014). Given the response of $\mathrm{THz}$ radiation to phonons in the crystal lattice, $\mathrm{THz}$ has been used in several cases to study crystalline cellulose; in one case, the CrI of microcrystalline cellulose (MCC) was determined by THz-TDS combined with a partial least squares (PLS) model (Vieira and Pasquini 2014), and the capability to distinguish between cellulose crystalline forms of $\mathrm{I}_{\alpha}$ and $\mathrm{I}_{\beta}$ was detected (Wang et al. 2020).

This study is a follow-up of a previous research (Wang et al. 2020). We continuously explored the possibility of THz-TDS in cellulose research. THz-TDS was used to investigate the cellulosebased materials, including MCC and wood, both ball milled at different times, and pseudo-wood which was a mixture of MCC and lignin. All materials showed a peak at $3.04 \mathrm{THz}$ of the mass absorption coefficient spectra, and the peak intensity decreased as the crystallinity decreased by ball milling. Further, the mass absorption coefficient spectra of $2.79 \mathrm{THz}$ to $3.32 \mathrm{THz}$ were cut out and 
detrended by subtraction of a baseline. The integrated intensity of the detrended spectra showed a correlation with the mass fraction of lignin of the pseudo-wood, and ball milling time of MCC and wood. Different from determination of the CrI of cellulose by deconvolution of the XRD pattern, the processing of $\mathrm{THz}$ mass absorption coefficient spectra was relatively simple. Moreover, the integrated intensity of $2.79 \mathrm{THz}$ to $3.32 \mathrm{THz}$ was normalized to $0-1$ by a min-max algorithm, and it can be regarded as a new "index" for evaluation of the cellulose crystallinity.

\section{Experimental}

\section{Sample preparation}

To obtain cellulose and wood powder with different CrI, the MCC powder (EMD Millipore 1.02331.0500) were ball milled with a benchtop ball mill (AV-2, Asahi Rika Factory. Ltd) at 200 rpm using ceramic spheres and jars for $0,12,24,48,72$, and 144 hours. An air-dried softwood, Hinoki cypress (Chamaecyparis obtuse), was first crushed by a rotary crusher and sieved with 10 US standard mesh to collect wood powder with a particle size of $200 \mu \mathrm{m}$. The wood powder was then ball milled for $0,6,12,24,32,48,72$, and 144 hours using the same procedure as the MCC powder.

2 The pseudo-wood powder was a mixture of MCC and organic-solvent lignin powder (Guangzhou Yinnovator Bio-tech Co., Ltd), where the lignin powder was extracted from Eucalyptus (Eucalyptus) by a high-concentration glacial acetic acid (>70\%). To obtain different CrIs, we used the following mass fractions of lignin in the powder: $0 \%, 25 \%, 50 \%, 75 \%$, and $100 \%$. The $100 \%$ organic-solvent lignin powder can be considered as a totally amorphous powder.

The prepared powders with different CrIs were collected at a mass of $0.075 \mathrm{~g}$ by an electronic balance $( \pm 0.0001 \mathrm{~g})$. All the powders were compressed into tablets with a diameter of $14 \mathrm{~mm}$ and a thickness of approximately $0.35 \mathrm{~mm}$ using a hand-pressing tableting kit (IMC-180C, Imoto Machinery Co., LTD). Three tablets were prepared for each powder. The thickness of these tablet samples was measured using a micrometer $( \pm 0.001 \mathrm{~mm})$. 


\section{XRD and THz-TDS measurement}

The XRD measurements of all the tablet samples were performed with $\mathrm{Cu}-\mathrm{K} \alpha$ radiation $(\lambda=$ $0.1542 \mathrm{~nm}$ ) using an X-ray diffractometer (Ultima IV, Rigaku) at a voltage of $40 \mathrm{kV}$ and a current of $40 \mathrm{~mA}$. Diffractograms were recorded from $5^{\circ}$ to $40^{\circ}$, where the scan range included the peak of the main crystal lattice of cellulose and wood. The scan speed was set to $5^{\circ} \min ^{-1}$, and the sampling step was $0.05^{\circ}$. The background diffractogram was obtained from an empty sample holder.

The THz transmission spectra of all the tablet samples were measured by using a Tera ProspectorKit model (NIPPO PRECISION Co., Ltd.), and the reference signals were obtained by measurement of air before and after the sample measurement. The THz beam was horizontally polarized with the bandwidth from about $0.1 \mathrm{THz}$ to $4.00 \mathrm{THz}$, and the spectral resolution was $0.02 \mathrm{THz}$, corresponding to the inverse of the temporal scan range $(50 \mathrm{ps})$. The diameter of the $\mathrm{THz}$ beam spot on the sample was around $3 \mathrm{~mm}$. Each measurement was recorded by averaging 100 scans to improve the signal-to-noise ratio. For reproducibility, all measurements were conducted thrice. To avoid the influence of water vapor on the measurement caused by $\mathrm{THz}$ absorption, we placed the whole THz optical system in an almost-closed acrylic box that was continuously filled with dry air to ensure the stability of humidity. All samples were placed in the box for 24 hours before measurement to balance the ambient humidity. When the samples were placed in the box, dry airflow was filled into the box until all the THz measurements were completed.

\section{Results and Discussion}

\section{Calculation of Crl with XRD pattern}

The original XRD pattern was cut out with a scattering range from $10^{\circ}$ to $40^{\circ}$ of pseudo-wood, MCC, and wood samples, as shown in Fig. 1(a), 1(b), and 1(c), respectively. Gradient colors were used to express changes in the ball milling time of samples and mass fractions of lignin. Background subtraction and baseline correction were performed on all original XRD patterns before further calculation. The background pattern was obtained as mentioned in the experimental section. The 
109 After background subtraction and baseline correction, the corrected XRD patterns can be

110 considered as composites of an amorphous intensity curve and five main crystalline peaks for the $\mathrm{I}_{\beta}$

111 type cellulose, a dominant type in vascular plants such as wood. The five main crystalline peaks had 112 Miller indices of (1 $\overline{1} 0),(110),(102),(200)$, and (004). The amorphous intensity curve was 113 determined by two different methods (Park et al. 2010), which will be discussed in detail later.

114 The deconvolution of all the crystalline peaks was carried out with a curve-fitting process using 115 a pseudo-Voigt profile, which is a linear combination of a Gaussian curve and a Lorentzian curve 116 as shown respectively in the following equations:

$$
\begin{gathered}
I_{G}(2 \theta)=I_{\text {max }} \cdot \exp \left\{-4 \ln (2) \cdot\left(\frac{2 \theta-2 \theta_{\max }}{\beta}\right)^{2}\right\} \\
I_{L}(2 \theta)=I_{\max }\left\{1+4\left(\frac{2 \theta-2 \theta_{\max }}{\beta}\right)^{2}\right\}^{-1} \\
I_{P V}(2 \theta)=\mu I_{L}+(\mu-1) I_{G}
\end{gathered}
$$

where $I_{\max }$ is the intensity of peaks, $2 \theta_{\max }$ is the peak position, and $\beta$ is the full width at half maximum (FWHM); $I_{G}(2 \theta)$ and $I_{L}(2 \theta)$ are the Gaussian and Lorentzian curves, respectively (de Keijser et al. 1983; Wada et al. 1997).

123 The positions $(2 \theta)$ of the crystalline peaks were fixed at $14.9^{\circ}, 16.7^{\circ}, 20.5^{\circ}, 22.6^{\circ}$, and $34.5^{\circ}$, 124 corresponding to the Miller indices of (11̄0), (110), (102), (200), and (004), respectively. The other 125 parameters of FWHM, the peak intensity, and coefficient $\mu$ were all determined by the curve-fitting 126 process.

127 In this study, the amorphous intensity curve was first fitted as a pseudo-Voigt profile, as shown 128 in Fig. 2(b), the same fitting process as that of other crystalline peaks. Given that recent research 129 showed that the maximum of the amorphous intensity curve is slightly more than $20^{\circ}$, therefor, the 130 peak position $(2 \theta)$ of the amorphous intensity curve was fixed at $20.6^{\circ}$ (Yao et al. 2020 ), where the 131 position was very close to the (102) crystalline peak, and the influence caused by this will be 132 discussed in detail later. 
134 was used in this study (Yao et al. 2020). The amorphous intensity curve was determined by fitting

135 the averaged XRD pattern of three $100 \%$ organic-solvent lignin samples using a 7th-order Fourier

136 series as shown in Fig. 2(c). The obtained Fourier series model can be considered as the basis

137 function of the amorphous intensity curve. The XRD patterns of other different samples only

138 showed the difference in intensity. The intensity can be adjusted by multiplying this Fourier series

139 by a coefficient $k$ in the deconvolution of XRD patterns:

$$
f(2 \theta)=k \cdot\left\{a_{0}+\sum_{i=1}^{7} a_{i} \cos (2 \theta \cdot w)+b_{i} \cos (2 \theta \cdot w)\right\}
$$

141 Supplementary Information provides the details of the fitting of the Fourier series of $100 \%$ 142 organic-solvent lignin samples.

143 The deconvolution results were evaluated with a coefficient of determination $R^{2}$ :

$$
R^{2}=1-\frac{\sum_{i=1}^{n}\left(X_{i}-Y_{i}^{\prime}\right)^{2}}{\sum_{i=1}^{n}\left(X_{i}-\bar{X}\right)^{2}}
$$

where $X_{i}$ is the intensity after background subtraction and baseline correction, $Y_{i}$ is the fitted

146 intensity, and $\bar{X}$ is the average of intensities. Table 1 summarizes the calculated $R^{2}$ of the pseudo-

147 wood fitted by two different amorphous intensity curves, and Supplementary Information

148 summarizes the $R^{2}$ values of other samples. As shown in Table 1, the curves fitted by both methods

149 showed good $R^{2}$, which indicates that the curve-fitting of the two methods was credible.

150 Table 1 Calculated $R^{2}$ values of the pseudo-wood fitted by two different amorphous intensity curves

\begin{tabular}{cccccc}
\hline Amorphous curve & \multicolumn{5}{c}{ Volume fraction of lignin } \\
\hline pseudo-Voigt & $0 \%$ & $25 \%$ & $50 \%$ & $75 \%$ & $100 \%$ \\
\hline \multirow{2}{*}{$R^{2}$} & 0.992 & 0.993 & 0.996 & 0.993 & 0.909 \\
& 0.990 & 0.987 & 0.987 & 0.989 & 0.960 \\
\hline & 0.982 & 0.996 & 0.996 & 0.994 & 0.898
\end{tabular}

Fourier series

$\begin{array}{llllll} & 0.991 & 0.993 & 0.997 & 0.994 & 0.910 \\ R^{2} & 0.988 & 0.986 & 0.988 & 0.991 & 0.962 \\ & 0.979 & 0.996 & 0.996 & 0.995 & 0.902\end{array}$

151 Once the peaks were deconvoluted by the curve-fitting process, the CrI was determined by the

152 following equation:

$$
C I=\frac{S_{C r}}{S_{C r}+S_{A m}}
$$


where $S_{C r}$ and $S_{A m}$ are the sum of the integrated intensity of five crystalline peaks and the integrated

155 intensity of amorphous intensity curve, respectively.

156 As shown in Fig. 2(b) and 2(c), the amorphous intensity curve fitted with a pseudo-Voigt profile

157 and a Fourier series, respectively, gave different deconvolution results. The most evident intensity

158 of the (102) peak in Fig. 2(b) was almost invisible, since the peak position of (102) peak was fixed

159 at $20.5^{\circ}$ and the amorphous peak was fixed at $20.6^{\circ}$, the contribution of the (102) peak was almost

160 systematically ignored in the fitting process. On the other hand, the (102) peak can be clearly

161 observed in Fig. 2(c). Thus, the CrI calculated with a pseudo-Voigt profile amorphous intensity

162 curve was smaller than that calculated with a Fourier series amorphous intensity curve on average.

163 Fig. 3 shows the CrI calculated from the different methods. In the figure, the amorphous curve

164 for (a) pseudo-wood, (b) MCC, and (c) wood was fitted by pseudo-Voigt profile, whereas that for

165 (d) pseudo-wood, (e) MCC, and (f) wood was fitted by Fourier series. As shown in Fig. 3, two

166 different fittings gave a similar crystallinity, whereas for the pseudo-wood, the crystallinity

167 decreased from about $85 \%$ to about $5 \%$ with the increase in mass fraction of lignin. For MCC and

168 wood, the crystallinity decreased from about $90 \%$ to $60 \%$ and $80 \%$ to $40 \%$ with the increase in ball-

169 milling time, respectively.

170 As shown in Fig. 3(a) and 3(d), for the pseudo-wood, the samples with a 25\% mass fraction of

171 organic-solvent lignin showed a similar crystallinity with the samples without organic-solvent lignin

172 (100\% MCC samples). A non-linear correlation was observed between the $\mathrm{CrI}$ and mass fraction of

173 lignin, where the CrI decreased sharply when the mass fraction of lignin was over 50\%. Regardless

174 of the fitting method used to obtain the curve, the sample that had $100 \%$ organic-solvent lignin,

175 which was supposed to have no crystalline region, still showed a crystallinity of about 5\%. The

176 crystallinity for MMC and wood showed a similar downtrend. In general, the concentration of

177 cellulose in different species of wood is about 30\% to 40\% (PETTERSEN 1984). However, in this

178 study, for most of the ball-milling times, the CrI of wood was about $10 \%$ lower than that of MCC.

179 For the same type of samples, the changes in the CrI calculated from the XRD patterns was easy to

180 observe and evaluate. However, when the cellulose concentration of wood was about $30 \%$ to $40 \%$,

181 the original wood samples still showed a relatively similar CrI compared with that of the original 
182 MCC samples. Thus, accurate comparison of the differences in CrIs between various types of

183 samples using the same standard can be a challenge. Table 2 summarizes the detailed crystallinity

184 of the pseudo-wood sample calculated from the two curve-fittings and the crystallinity calculated

185 from the THz mass absorption coefficient spectrum, which will be discussed in detail in the next

186 section. Supplementary Information summarizes the crystallinities of other samples.

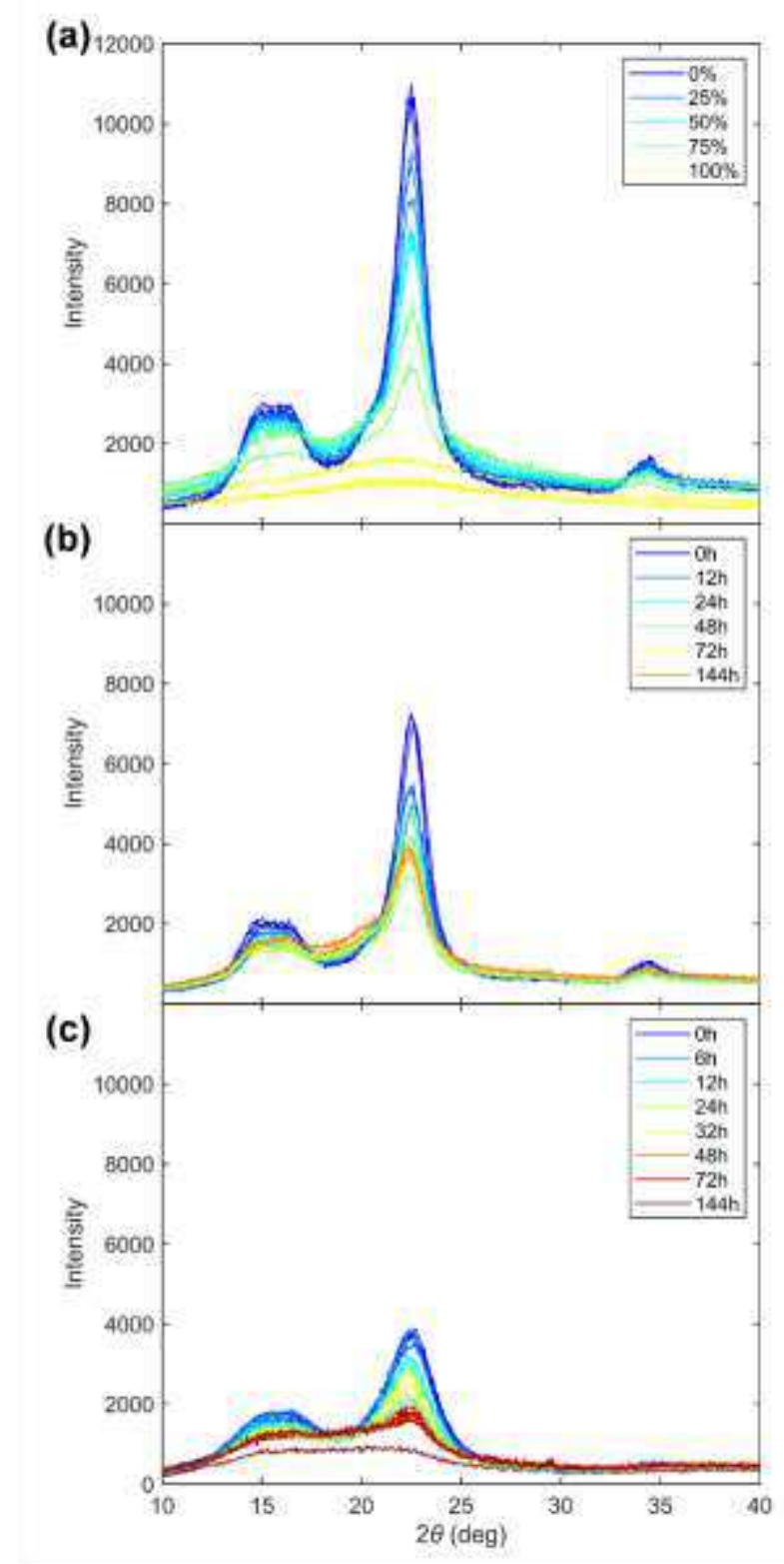

188 Fig.1 Original XRD patterns of (a) pseudo-wood with a decreased mass fraction of organic-solvent

189 lignin, (b) MCC, and (c) wood samples. 
(a)
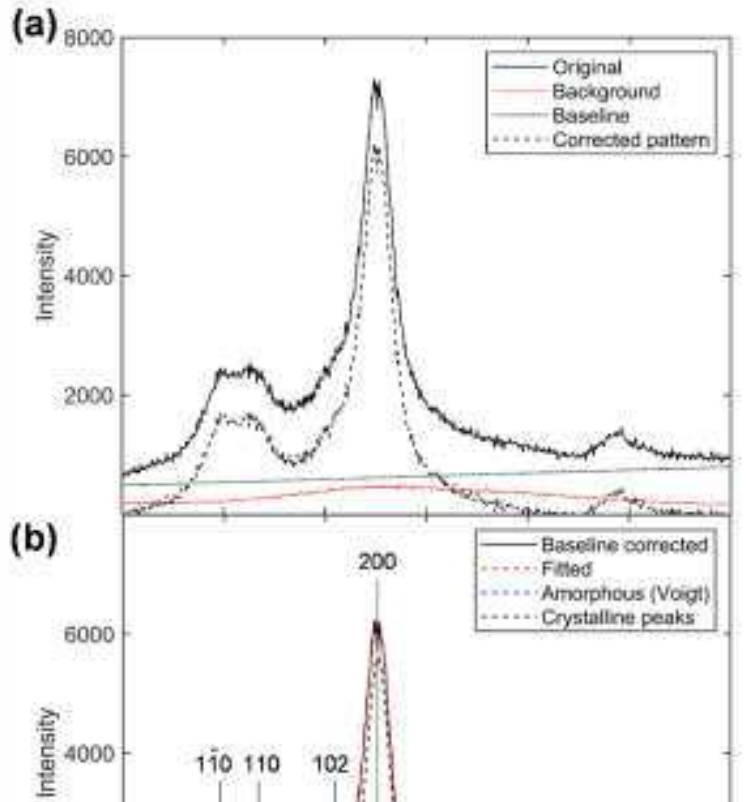

190

(c)

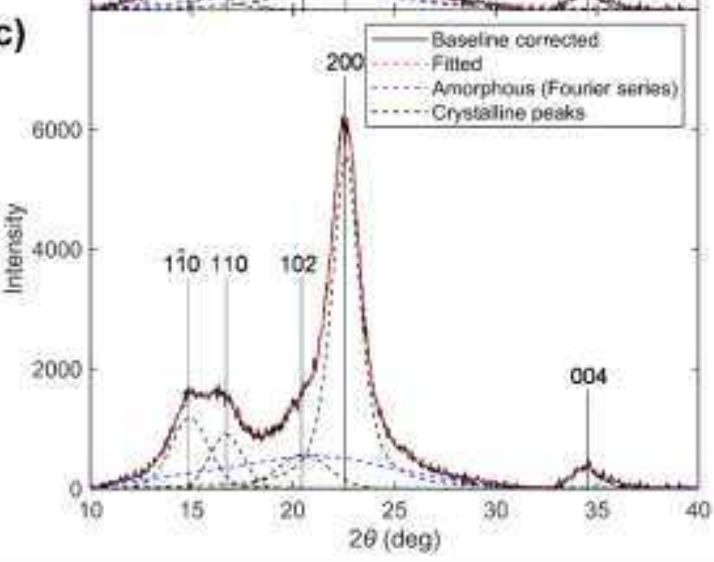

191 Fig. 2 Curve-fitting process of a pseudo-wood with 50\% lignin. (a) Background subtraction and

192 baseline correction, (b) deconvolution of peaks by using a pseudo-Voigt profile only, and (c) the

193 deconvolution of peaks where the crystalline peaks fitted by using a pseudo-Voigt profile; the

194 amorphous curve was fitted by a 7th-order Fourier series model. 
(a)

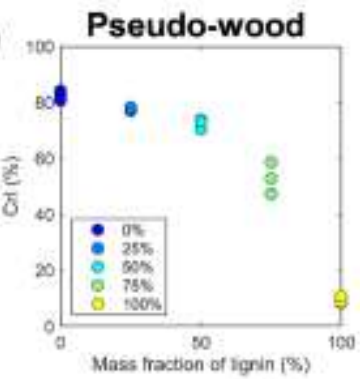

(d)

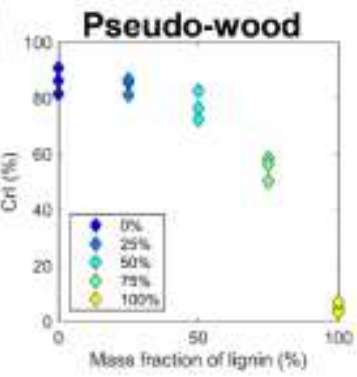

(b)

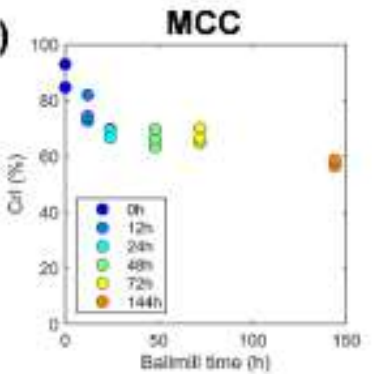

(e)

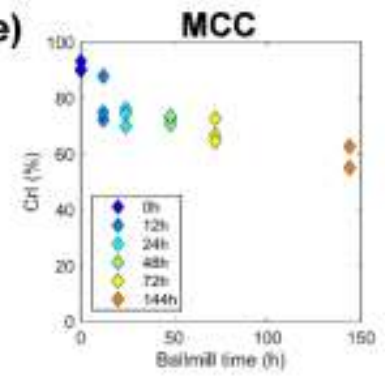

(c)

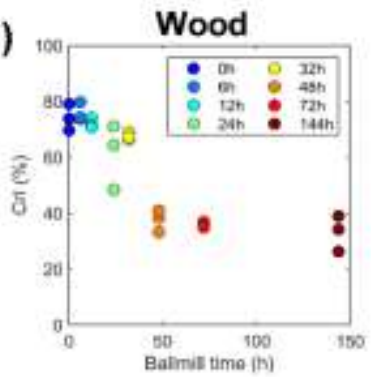

(f)

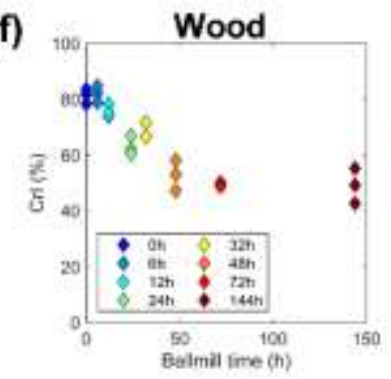

195

Fig.3 CrI calculated from two different curve-fitting processes of the amorphous curve, where (a) - (c)

with circle markers were fitted by a pseudo-Voigt profile, and (d) - (e) with diamond markers were fitted by a 7th-order Fourier series

\section{Evaluation of $\mathrm{Crl}$ by $\mathrm{THz}$ mass absorption coefficient spectra}

The measured $\mathrm{THz}$ time-domain signal was Fourier transformed into the frequency domain. Given that the samples used in this study were all "optically thick samples," where the backward and forward reflections in the sample (Fabry-Pérot effect) can be ignored (Duvillaret et al. 1996), further to correct the possible inhomogeneity of hand-made samples, the mass absorption coefficient $\alpha$ that also considers the influence of density was calculated using the following equations:

$$
\alpha=\frac{1}{\rho L} \ln \left(\frac{R_{r}}{R_{s}}\right)
$$

where $\rho$ is the sample density, $L$ is the sample thickness, $R_{r}, R_{s}$ are the phase and amplitude in the frequency domain of the reference and measured samples, respectively, and (Kore and Pawar 2014).

Fig. 4 (a), 4(b), and 4(c) showed the original THz mass absorption coefficient spectra from 0.2 THz to $4 \mathrm{THz}$ of the pseudo-wood, MCC, and wood, respectively. The same gradient colors as that in Fig. 1 were used. The calculated mass absorption coefficient spectra were all first corrected by baseline fluctuations with a standard normal variate algorithm (SNV) and then smoothed by the 
212 application of a Savitzky-Golay filter with a second-order polynomial and fifteen smoothing points,

213 as shown in Fig. 4(d) - 4(f). The pseudo-wood and MCC samples showed absorption peaks at

214 around $2.1 \mathrm{THz}$, and these peaks gradually decreased with the increase in mass fraction of lignin or

215 ball-milling time, whereas at this frequency position, no evident absorption peaks of wood samples

216 were observed. Previous research discovered that the absorption peaks at $2.1 \mathrm{THz}$ correlated with

217 crystalline structures, where the $\mathrm{I}_{\beta}$ - dominant and $\mathrm{I}_{\alpha}$-rich types showed absorption peaks at 2.11 and

$2182.38 \mathrm{THz}$, respectively. Furthermore, the intensity of the peaks here was correlated with the fraction

219 of $\mathrm{I}_{\alpha}$. The larger the fractions of $\mathrm{I}_{\alpha}$, the stronger the absorption at $2.38 \mathrm{THz}$. On the contrary, the

220 smaller the fraction of $\mathrm{I}_{\alpha}$, the stronger the absorption at $2.11 \mathrm{THz}$ (Wang et al. 2020). Similar to the

221 case of wood samples, the absorption peaks of pseudo-wood samples with the mass fraction over

$22250 \%$ of organic-solvent lignin were difficult to observe at around $2.1 \mathrm{THz}$, whereas the cellulose

223 concentration of wood samples was about 30\%. Therefore, no evident absorption peak, such as that

224 of MCC samples at around $2.1 \mathrm{THz}$ can be observed. This phenomenon might have been caused by

225 the low cellulose concentration (about 30\%) of wood. The thickness of cellulose samples used in

226 the previous research was about $0.5 \mathrm{~mm}$ to $1 \mathrm{~mm}$ (Wang et al. 2020), which was thicker than that

227 of the samples in this study. Given that the absorption around $3 \mathrm{THz}$ is correlated with the CrI of

228 cellulose (Vieira and Pasquini 2014), the samples were made thin in this study to improve the signal-

229 to-noise ratio to investigate further the changing absorption peaks around $3 \mathrm{THz}$. However, this

230 condition may lead to difficult observation of the small absorption peaks around $2 \mathrm{THz}$.

231 As shown in Fig. 4(a) - 4(f), all types of samples expressed a relatively strong absorption at 3.04

$232 \mathrm{THz}$ without a peak shift. ling time. The mass absorption coefficient spectra of wood showed a

233 relatively small intensity compared with other samples. To further study this absorption peak, we

234 cut out the mass absorption coefficient spectra from the range of $2.79 \mathrm{THz}$ to $3.32 \mathrm{THz}$ and then

235 subtracted the baseline that was fitted as a first-order polynomial. Fig. 4(g), 4(h), and 4(i) show the

236 baseline corrected mass absorption coefficient spectra of the pseudo-wood, MCC, and wood,

237 respectively. The integrated intensities of all the detrended mass absorption coefficient spectra were

238 calculated, and their correlations with the mass fraction of lignin and ball-milling time are plotted

239 in Fig. 5(a), 5(b), and 5(c). 
241 the mass absorption coefficient spectra from $2.79 \mathrm{THz}$ to $3.32 \mathrm{THz}$ showed a linear correlation with

242 the mass fractions of lignin. Given that the mass fraction of lignin was adjusted to increase linearly

243 (+25\% each time) during the sample preparation process, the correlation obtained from $\mathrm{THz}$ mass

244 absorption coefficient spectra is more reasonable than that obtained from XRD patterns. The

245 correlations between the ball-milling time with the integrated intensity of the MCC and wood

246 samples showed a similar trend with that of the crystallinity obtained from the XRD patterns.

247 However, the values of $144 \mathrm{~h}$ ball-milled MCC samples decreased by about $50 \%$ compared with

248 that of the original MCC samples, whereas the CrI of the 144 hours ball-milled MCC samples

249 calculated from XRD patterns decreased by about $25 \%$. Furthermore, the original wood samples

250 without ball milling showed relatively small values compared with the original MCC samples. By

251 contrast, the CrI of the original wood samples calculated from the XRD pattern showed no evident

252 difference with that of original MCC samples. The integrated intensities of the original wood

253 samples were about $30 \%$ those of the original MCC samples, thus matching with the approximate

254 concentration of cellulose in wood (about 30\%). Table 2 summarizes the detailed integrated

255 intensities of the pseudo-wood samples and the CrI calculated by two curve-fittings from the XRD 256 patterns.

257 The integrated intensities of all samples were normalized to the range of 0 to 1 by the min-max 258 algorithm, and the results are presented in Fig. 4(d), 4(e), and 4(f). After this process, the normalized 259 value can be considered as an "index" that can be used to evaluate crystallinity. Different from the 260 CrI, which is a relative value calculated from XRD patterns, an assumption was easily reached from 261 the above results, that is, the THz mass absorption coefficient spectra may reveal the absolute mass 262 fractions of crystalline cellulose in all the samples used in this study. Given that the peaks of the 263 mass absorption coefficient in the THz region did not shift with the changes the type of samples or 264 ball-milling time and mass fraction of lignin, only the intensity changed. Moreover, the THz spectra 265 contain rich physical and chemical information, such as the phonon frequency of crystal lattices, 266 which provides a possibility for the comparison of the mass fraction of crystalline cellulose between 267 different cellulose-based materials. However, the energy corresponding to $3 \mathrm{THz}$ is $0.012 \mathrm{eV}$, which 
268 is relatively small compared with the stabilization energy of hydrogen bonding in cellulose $\mathrm{I}_{\beta}$, for 269 example, the intrasheet O6-H...O3 H-bonds with the stabilization energy of about $0.87 \mathrm{eV}$ 270 (Parthasarathi et al. 2011). Without further research based on the theoretical proof level such as the 271 quantum mechanical calculation using density-functional theory (DFT), the assignments of 272 absorption peaks at $3.04 \mathrm{THz}$ are still uncertain.
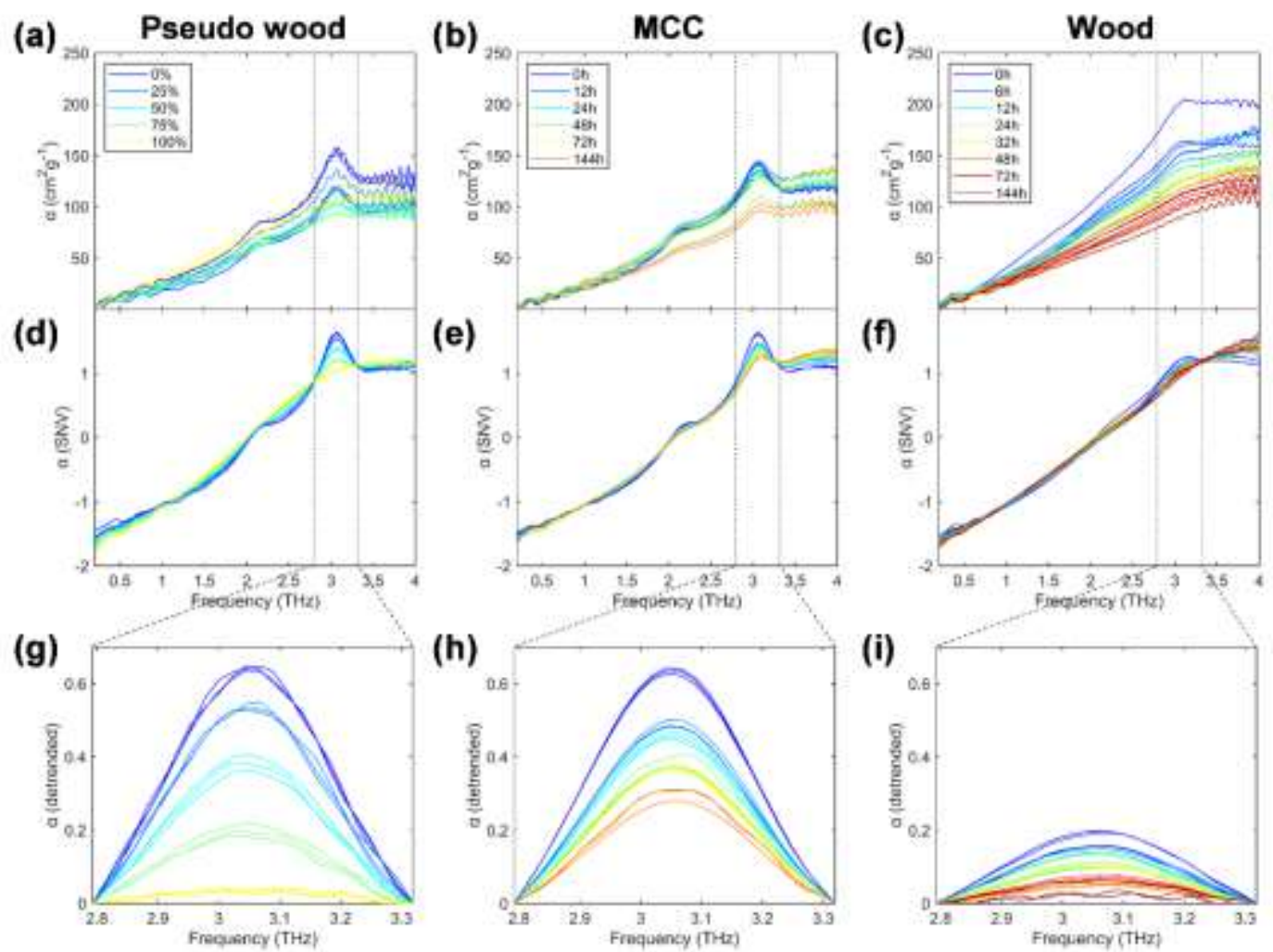

274 Fig. 4 Original THz mass absorption coefficient spectra of (a) pseudo-wood, (b) MCC, and (c) wood

275 samples. (d), (e), and (f) SNV and smoothed mass absorption coefficient spectra. (g), (h), and (i)

276 Detrended mass absorption coefficient spectra obtained by subtraction of the baseline at the frequency 

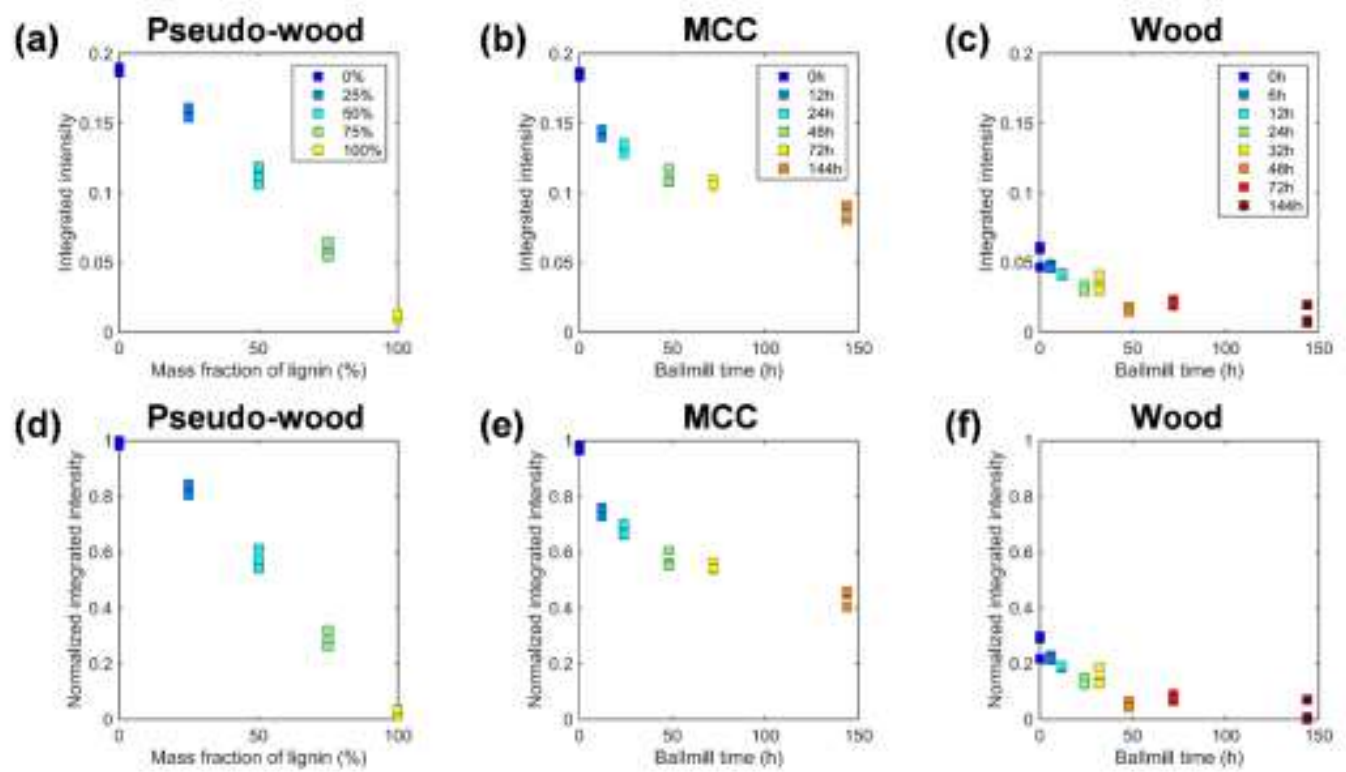

279 Fig. 5 Integrated intensity calculated from the detrended mass absorption coefficient spectra from 2.79

280 THz to $3.32 \mathrm{THz}$ of (a) pseudo-wood, (b) MCC, and (c) wood samples. (d), (e), and (f) Normalized

281 integrated intensity by min-max algorithm

282 Table 2 CrI of the pseudo-wood calculated from XRD patterns and the integrated intensity obtained from

$283 \quad 2.79 \mathrm{THz}$ to $3.32 \mathrm{THz}$ mass absorption coefficient spectra

\begin{tabular}{|c|c|c|c|c|c|}
\hline \multirow[t]{2}{*}{ Calculation method } & \multicolumn{5}{|c|}{ Volume fraction of lignin } \\
\hline & $0 \%$ & $25 \%$ & $50 \%$ & $75 \%$ & $100 \%$ \\
\hline XRD with pseudo-Voigt Am & 84.270 & 77.024 & 73.808 & 47.351 & 8.393 \\
\hline \multirow[t]{2}{*}{$\mathrm{CrI}(\%)$} & 82.361 & 77.163 & 70.362 & 58.641 & 8.586 \\
\hline & 80.704 & 78.268 & 73.242 & 52.810 & 10.794 \\
\hline \multicolumn{6}{|l|}{ XRD with Fourier series Am } \\
\hline \multirow[t]{3}{*}{$\operatorname{CrI}(\%)$} & 90.870 & 85.692 & 76.463 & 50.256 & 4.966 \\
\hline & 86.272 & 81.236 & 72.402 & 58.293 & 3.296 \\
\hline & 81.576 & 86.667 & 82.702 & 56.254 & 7.075 \\
\hline \multicolumn{6}{|c|}{ Detrended THz mass absorption coefficient } \\
\hline \multirow[t]{3}{*}{ integrated intensity } & 0.171 & 0.132 & 0.095 & 0.051 & 0.011 \\
\hline & 0.142 & 0.133 & 0.093 & 0.048 & 0.013 \\
\hline & 0.178 & 0.139 & 0.083 & 0.041 & 0.013 \\
\hline \multicolumn{6}{|l|}{ Min-maxed integrated intensity } \\
\hline & 0.957 & 0.734 & 0.518 & 0.261 & 0.027 \\
\hline & 0.789 & 0.740 & 0.506 & 0.246 & 0.039 \\
\hline & 1.000 & 0.774 & 0.448 & 0.206 & 0.044 \\
\hline
\end{tabular}




\section{Conclusions}

In this study, pseudo-wood, MCC, and wood samples were adjusted to different cellulose CrI by

287 changing the mass fraction of lignin of pseudo-wood samples and the ball-milling time of MCC and wood samples. The measured XRD patterns of all samples were deconvoluted by two different curve-fitting processes, in which one used a pseudo-Voigt profile, and the other used a 7th-order Fourier series to represent the amorphous intensity curve. Both methods gave good fitting results, and the calculated CrIs were close. All samples showed peaks at $3.04 \mathrm{THz}$ of the $\mathrm{THz}$ mass absorption coefficient spectra. The THz mass absorption coefficient spectra were pretreated by SNV to correct baseline fluctuations, smoothed by the application of a Savitzky-Golay filter with fifteen points, and then detrended by subtraction of the baseline from $2.79 \mathrm{THz}$ to $3.32 \mathrm{THz}$. The integrated intensities showed a similar changing trend with the CrIs obtained from XRD patterns. However, evident differences were observed. For the pseudo-wood, the correlation between the $\mathrm{CrI}$ and mass fraction of lignin were non-linear, whereas the integrated intensity of detrended $\mathrm{THz}$ mass absorption coefficient spectra decreased linearly with the increase in the mass fraction of lignin. The original wood sample without ball milling had an integrated intensity value that was about $30 \%$ of that in the original MCC sample, matching the cellulose concentration of wood. By contrast, the CrI of the original wood sample calculated from XRD patterns showed a similar value to that of the original MCC sample. Thus, based on the above results, THz mass absorption coefficient spectra may be used to evaluate the mass fraction of crystalline cellulose. Furthermore, after normalization

304 of the integrated intensity by a min-max algorithm to the range of 0 to 1 , the original MCC sample without lignin and the $100 \%$ lignin sample can be considered as a pair of standard samples 306 corresponding to 1 and 0 , respectively. All the cellulose crystallinity can be evaluated in this range.

307 However, the assignments of absorption peaks of cellulose at $3.04 \mathrm{THz}$ are still uncertain. Numerous 308 optional functions that rely on the experience of analysts, such as pseudo-Voigt profile and Fourier series used in this study, are available for the deconvolution of XRD patterns. Meanwhile, the 
311 showed peaks at $3.04 \mathrm{THz}$ without a peak shift. The measurement of $\mathrm{THz}$ signal is quick, simple,

312 and safe and requires no sample pretreatment. Thus, THz-TDS has the potentiality of becoming a

313 new standard for the evaluation of cellulose crystallinity.

314 Acknowledgments The authors would like to express our grateful appreciation to Dr. Liu and Dr.

315 Wang for the supplement of the organic-solvent lignin samples. This work was supported by JSPS

316 KAKENHI Grant Number 16H02559. And the first author also thanks for the Mitsutani scholarship for

317 living support.

\section{Reference}

319 Braly LB, Liu K, Brown MG, et al. (2000) Terahertz laser spectroscopy of the water dimer

320 intermolecular vibrations. II. (H2O)2. J Chem Phys 112:10314-10326.

321 de Keijser T, Mittemeijer EJ, Rozendaal HCF (1983) The determination of crystallite-size and lattice-

322 strain parameters in conjunction with the profile-refinement method for the determination of crystal

323 structures. J Appl Crystallogr 16:309-316.

324 del Cerro DR, Koso T V., Kakko T, et al. (2020) Crystallinity reduction and enhancement in the

325 chemical reactivity of cellulose by non-dissolving pre-treatment with tetrabutylphosphonium acetate.

326 Cellulose 27:5545-5562.

327 Density ANDC, Kellogg RM, Sastr CBR, Ivellwood RW (1975) Relationships Between Cell-Wall

328 Composition. 7:170-177

329 Duvillaret L, Garet F, Coutaz JL (1996) A reliable method for extraction of material parameters in

330 terahertz time-domain spectroscopy. IEEE J Sel Top Quantum Electron 2:739-745.

331 French AD (2014) Idealized powder diffraction patterns for cellulose polymorphs. Cellulose 21:885-

332896

333 French AD, Santiago Cintrón M (2013) Cellulose polymorphy, crystallite size, and the Segal

334 Crystallinity Index. Cellulose 20:583-588.

335 Hall M, Bansal P, Lee JH, et al. (2010) Cellulose crystallinity - A key predictor of the enzymatic

336 hydrolysis rate. FEBS J 277:1571-1582. 
337 Hermans PH, Weidinger A (1948) Quantitative x-ray investigations on the crystallinity of cellulose

338 fibers. A background analysis. J Appl Phys 19:491-506.

339 Himmel ME, Ding S, Johnson DK, Adney WS (2007) Biomass recalcitrance: engineering plants and

340 enzymes for biofuels production. Science (80- ) 315 (5813):804-808.

341 Hindeleh AM, Johnson DJ (1972) Crystallinity and crystallite size measurement in cellulose fibres: 1.

342 Ramie and Fortisan. Polymer (Guildf) 13:423-430.

343 Inagaki T, Ahmed B, Hartley ID, et al. (2014a) Simultaneous prediction of density and moisture

344 content of wood by terahertz time domain spectroscopy. J Infrared, Millimeter, Terahertz Waves

$345 \quad 35: 949-961$.

346 Inagaki T, Hartley ID, Tsuchikawa S, Reid M (2014b) Prediction of oven-dry density of wood by time-

347 domain terahertz spectroscopy. Holzforschung 68:61-68.

348 Inagaki T, Siesler HW, Mitsui K, Tsuchikawa S (2010) Difference of the crystal structure of cellulose

349 in wood after hydrothermal and aging degradation: A NIR spectroscopy and XRD study.

350 Biomacromolecules 11:2300-2305.

351 Jörgensen L, Kiessling R, Lindstedt G, Kinell P-O (1950) Swelling and Heterogeneous Hydrolysis of

352 Cotton Linters and Wood Pulp Fibers Related to Their Fine Structure. Acta Chem. Scand. 4:185-199

353 Kataoka Y, Kondo T (1998) FT-IR microscopic analysis of changing cellulose crystalline structure

354 during wood cell wall formation. Macromolecules 31:760-764.

355 Kim SH, Lee CM, Kafle K (2013) Characterization of crystalline cellulose in biomass: Basic

356 principles, applications, and limitations of XRD, NMR, IR, Raman, and SFG. Korean J Chem Eng

$357 \quad 30: 2127-2141$.

358 Kore PS, Pawar PP (2014) Measurements of mass attenuation coefficient, effective atomic number and

359 electron density of some amino acids. Radiat Phys Chem 98:86-91.

360 Langan P, Sukumar N, Nishiyama Y, Chanzy H (2005) Synchrotron X-ray structures of cellulose I $\beta$

361 and regenerated cellulose II at ambient temperature and $100 \mathrm{~K}$. Cellulose 12:551-562.

362 Ling Z, Wang T, Makarem M, et al. (2019) Effects of ball milling on the structure of cotton cellulose.

363 Cellulose 26:305-328. 
365 Top Quantum Electron 14:180-190.

366 Nishiyama Y (2009) Structure and properties of the cellulose microfibril. J Wood Sci 55:241-249.

367 Nishiyama Y, Langan P, Chanzy H (2002) Crystal structure and hydrogen-bonding system in cellulose

368 I $\beta$ from synchrotron X-ray and neutron fiber diffraction. J Am Chem Soc 124:9074-9082.

369 Parthasarathi R, Bellesia G, Chundawat SPS, et al. (2011) Insights into hydrogen bonding and stacking 370 interactions in cellulose. J Phys Chem A 115:14191-14202.

371 PETTERSEN RC (1984) The Chemical Composition of Wood. In: The Chemistry of Solid Wood. pp $372 \quad 57-126$

373 Plusquellic DF, Siegrist K, Heilweil EJ, Esenturk O (2007) Applications of terahertz spectroscopy in 374 biosystems. ChemPhysChem 8:2412-2431.

375 Poletto M, Zattera AJ, Forte MMC, Santana RMC (2012) Thermal decomposition of wood: Influence

376 of wood components and cellulose crystallite size. Bioresour Technol 109:148-153.

377 Segal L, Creely JJ, Martin AE, Conrad CM. An Empirical Method for Estimating the Degree of

378 Crystallinity of Native Cellulose Using the X-Ray Diffractometer. Textile Research Journal.

379 Teeäär R, Serimaa R, Paakkarl T (1987) Crystallinity of cellulose, as determined by CP/MAS NMR

380 and XRD methods. Polym Bull 17:231-237.

381 Thygesen A, Oddershede J, Lilholt H, et al. (2005) On the determination of crystallinity and cellulose 382 content in plant fibres. Cellulose 12:563-576.

383 Updegraff DM (1969) Semimicro determination of cellulose inbiological materials. Anal Biochem $38432: 420-424$.

385 Vieira FS, Pasquini C (2014) Determination of cellulose crystallinity by terahertz-time domain 386 spectroscopy. Anal Chem 86:3780-3786.

387 Wada M, Okano T, Sugiyama J (1997) Synchrotron-radiated X-ray and neutron diffraction study of 388 native cellulose. Cellulose 4:221-232.

389 Wang H, Horikawa Y, Tsuchikawa S, Inagaki T (2020) Terahertz time-domain spectroscopy as a novel 390 tool for crystallographic analysis in cellulose. Cellulose 27:9767-9777. 
391 Wang H, Inagaki T, Hartley ID, et al. (2019) Determination of Dielectric Function of Water in THz

392 Region in Wood Cell Wall Result in an Accurate Prediction of Moisture Content. J Infrared,

393 Millimeter, Terahertz Waves 40:673-687.

394 Xie L, Yao Y, Ying Y (2014) The application of terahertz spectroscopy to protein detection: A review.

395 Appl Spectrosc Rev 49:448-461.

396 Yao W, Weng Y, Catchmark JM (2020) Improved cellulose X-ray diffraction analysis using Fourier 397 series modeling. Cellulose 27:5563-5579. 
Figures

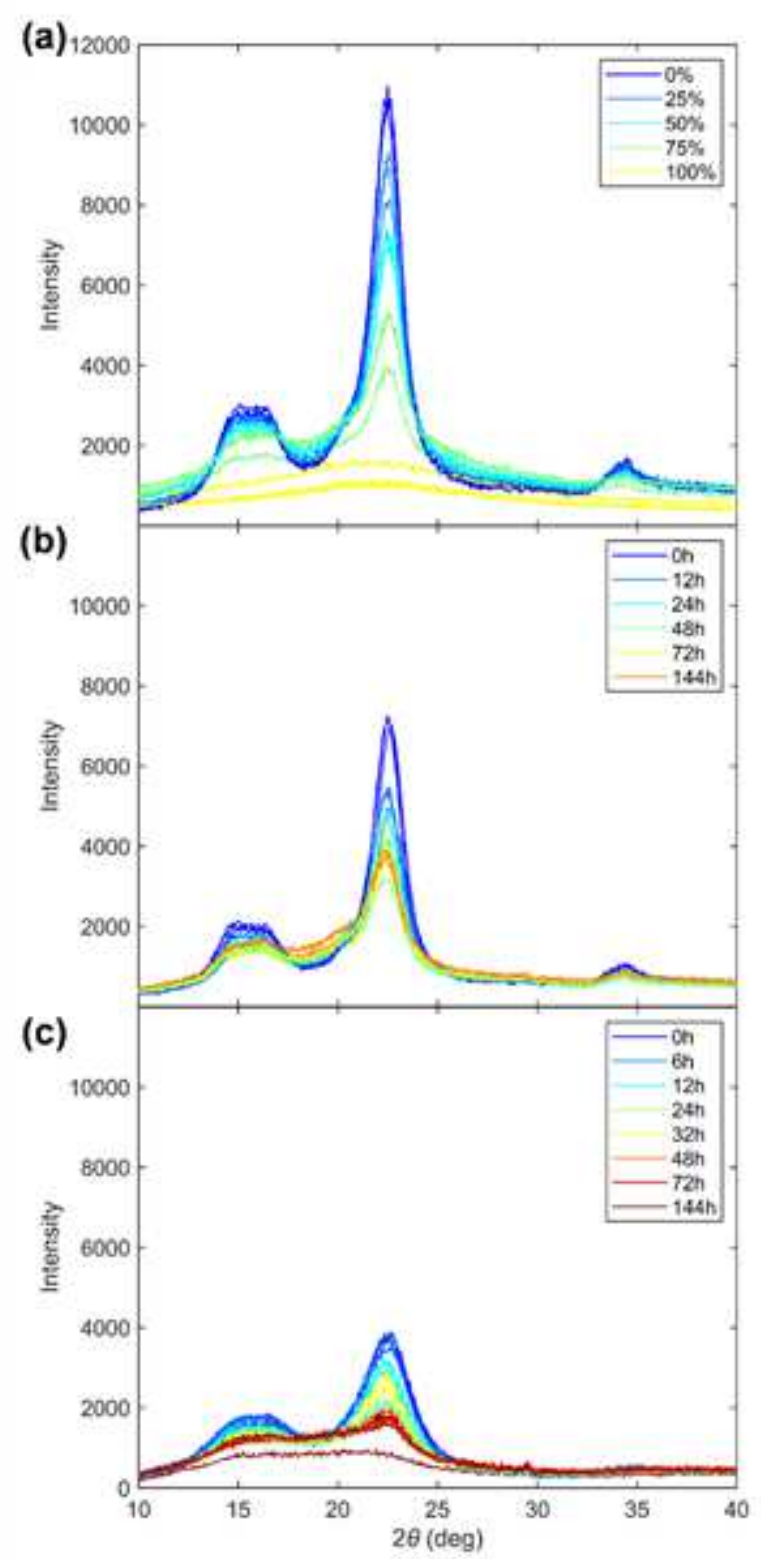

\section{Figure 1}

Original XRD patterns of (a) pseudo-wood with a decreased mass fraction of organic-solvent lignin, (b) MCC, and (c) wood samples. 


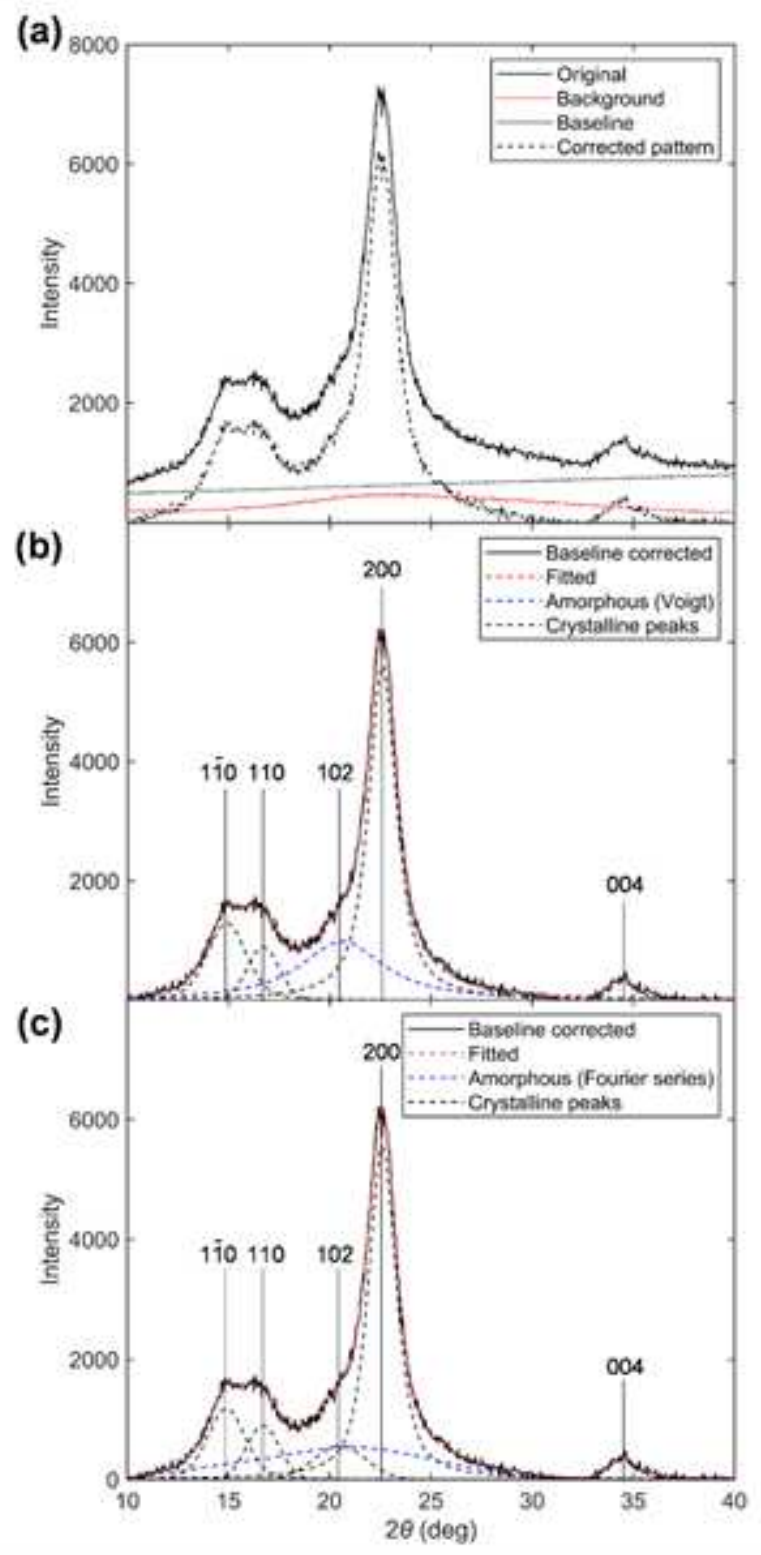

\section{Figure 2}

Curve-fitting process of a pseudo-wood with $50 \%$ lignin. (a) Background subtraction and baseline correction, (b) deconvolution of peaks by using a pseudo-Voigt profile only, and (c) the deconvolution of peaks where the crystalline peaks fitted by using a pseudo-Voigt profile; the amorphous curve was fitted by a 7th-order Fourier series model. 
(a)

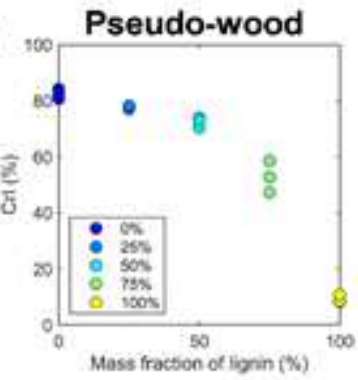

(d)

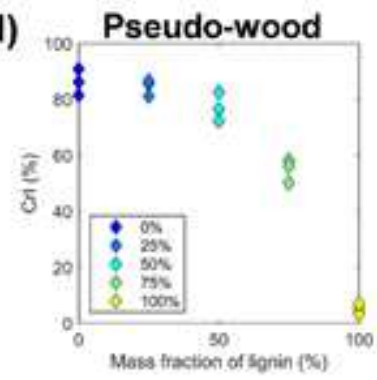

(b)

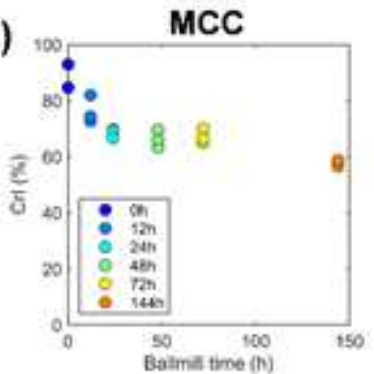

(e)

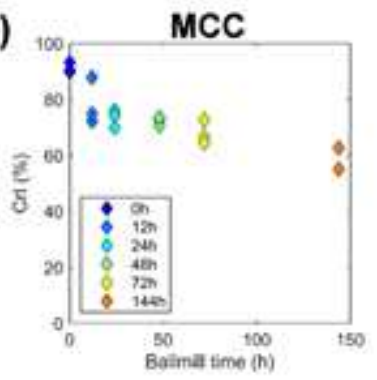

(c)

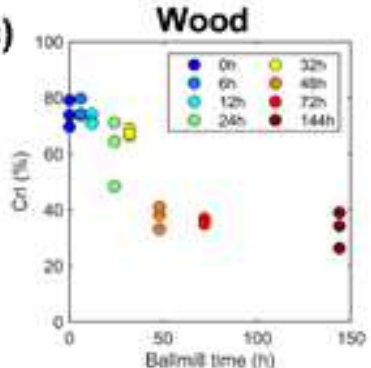

(f)

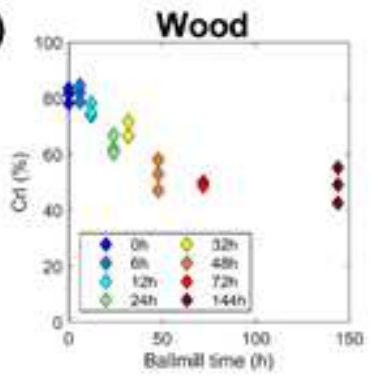

Figure 3

Crl calculated from two different curve-fitting processes of the amorphous curve, where (a) - (c) with circle markers were fitted by a pseudo-Voigt profile, and (d) - (e) with diamond markers were fitted by a 7th-order Fourier series
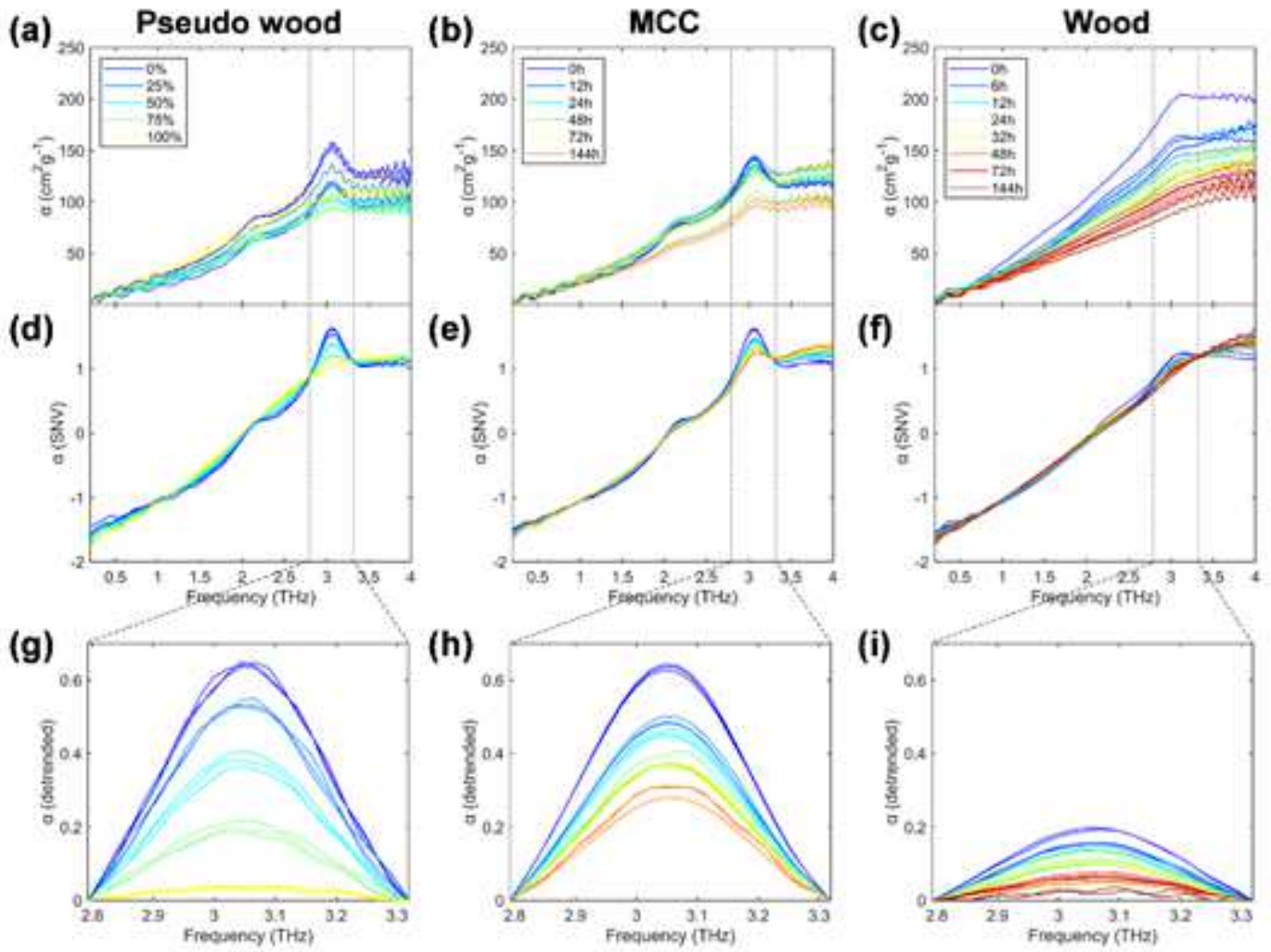

\section{Figure 4}

Original THz mass absorption coefficient spectra of (a) pseudo-wood, (b) MCC, and (c) wood samples. (d), (e), and (f) SNV and smoothed mass absorption coefficient spectra. (g), (h), and (i) Detrended mass 
absorption coefficient spectra obtained by subtraction of the baseline at the frequency range of $2.79 \mathrm{THz}$ to $3.32 \mathrm{THz}$
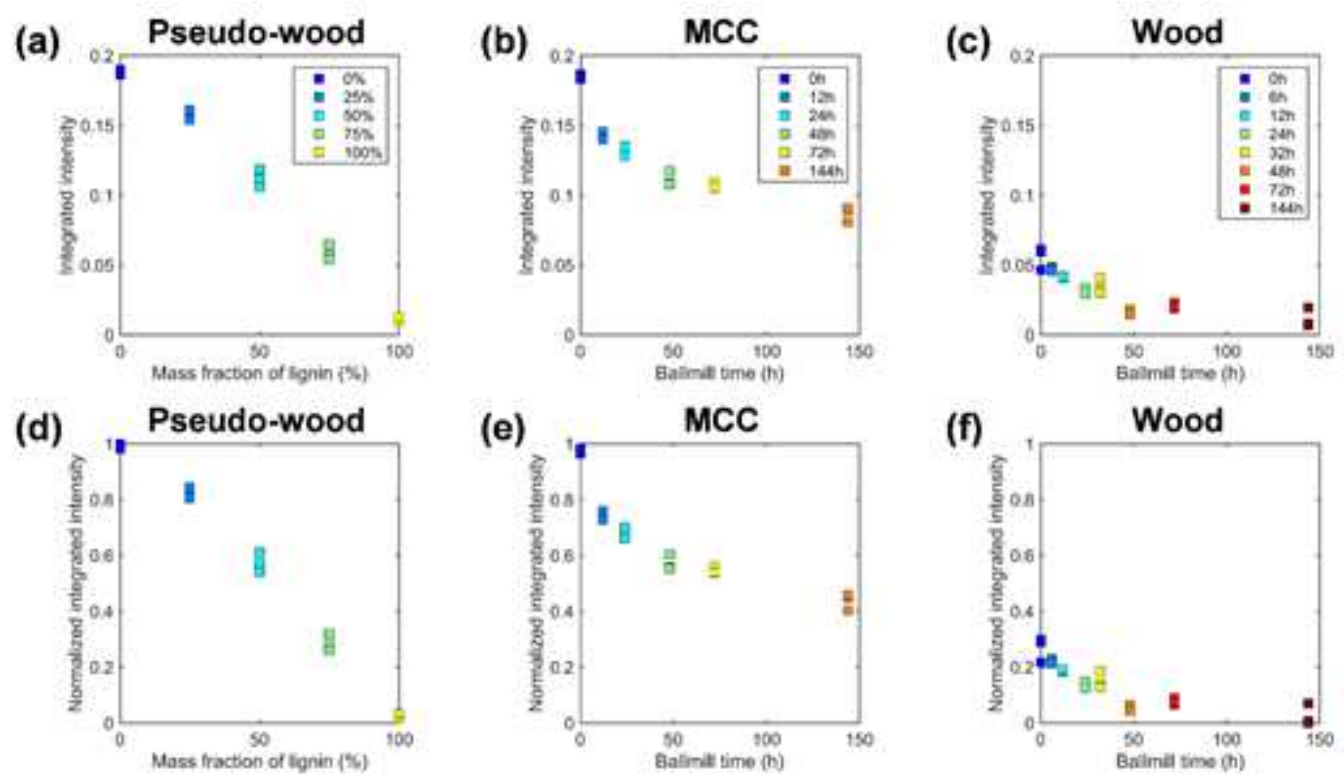

\section{Figure 5}

Integrated intensity calculated from the detrended mass absorption coefficient spectra from $2.79 \mathrm{THz}$ to 3.32 THz of (a) pseudo-wood, (b) MCC, and (c) wood samples. (d), (e), and (f) Normalized integrated intensity by min-max algorithm 